\title{
Glueballs and the universal energy spectrum of tight knots and links:
}

\author{
Roman V. Buniy and Thomas W. Kephart \\ Department of Physics and Astronomy, Vanderbilt University, Nashville, TN 37235, USA
}

\begin{abstract}
Systems of tightly knotted, linked, or braided flux tubes will have a universal mass-energy spectrum if the flux is quantized. We focus on a model of glueballs as knotted QCD flux tubes.
\end{abstract}

Plasma physics informs us that linked magnetic flux tubes are much more stable than an unknotted single loop 1]. Linked and knotted flux tubes carry topological quantum numbers, and one can think of a knot as a selflinked loop. Similar comments apply to braids.

Our interest will be in tubes carrying quantized flux in tight knot and link configurations. If the loops (tubes) have fixed uniform thickness and circular cross-section, then each knot and link has a completely specified length if the configuration is tight, i.e., is of the shortest length with the tubes non-overlapping and their cross-sections undistorted. If tubes have uniform cross sections, as can be approximately the case for many physical systems, then the length of the tight knot is proportional to the mass (or energy) of the knot. This, we claim, generates a universal mass (energy) spectrum for knotted/linked configurations of objects of this type. The lengths of tight knots were not studied until the mid-1990s [2], and only recently have accurate calculations of large numbers of tight knots [3] and links [4] become available. These results now make it possible to examine physical systems and compare them with the knot spectrum. We have examined the glueball spectrum of QCD 5], 6]. Glueballs 7] are likely to be solitonic states (See Ref. [5] for detailed references.) that are solutions to the QCD field equations. While QCD will be our main focus, there are many more systems where tight knots may play a role.

In order to decide if a system of flux tubes falls into the universal class of having a tight knot energy spectrum, we must first investigate the time scales involved. These are the lifetime of the soliton $\tau_{s}$ and the relaxation time $\tau_{r}$ necessary to reach the ground state of a knotted configuration (i.e., the tight knot state). The soliton lifetime (or the corresponding decay width $\Gamma_{s}=1 / \tau_{s}$ ) can depend on several factors. These include the effects of flux tube breaking, rearrangement, and reconnection. The partial width for flux tube breaking is non-zero if the production of particle/anti-particle pairs at the break point is energetically allowed, for example quark/anti-quark $(q \bar{q})$ pairs for color electric flux tubes. The partial widths can vary widely depending on the particle masses. Rearrangement is a quantum effect where, for example, in a linked double donut arrangement, the loops can tun-

\footnotetext{
*Talk given by TWK at Coral Gables 2003 in celebration of Paul Frampton's 60th birthday

${ }^{\dagger}$ Electronic address: roman.buniy@vanderbilt.edu

‡Electronic address: thomas.w.kephart@vanderbilt.edu
}

nel free of each other. Finally, reconnection is an effect where tubes break and re-attach in a different configuration. Such behavior has been seen in plasma physics and is of major importance in understanding a variety of astrophysical systems. All these processes change topological charge, and their partial widths compete more or less favorably with each other depending on the parameters that describe the system.

While no knot lengths have been calculated exactly, it is possible to calculate the exact lengths of an infinite number of links and braids [4]. For links, these calculations are possible in the case where individual elements of the link lie in planes. For braids, exact calculations are possible when the elements of the braid are either straight sections or where their centerlines follow helical paths. The shortest of all links, the double donut, is exactly calculable. The two elements lie in perpendicular planes and are tori of equal length. The shortest non-trivial braid is a helically twisted pair. "Weyl's tube formula" [8, 9] states that for a tube of constant crosssection $\sigma$ normal to a path of length $l$, the volume of the tube in flat $3 \mathrm{D}$ is just $V_{T}=l \sigma$. If we have an analytic form for the path and a circular cross-section we can find $V_{T}$. This leads to the class of exactly calculable links and braids, but since there are no known analytic forms for the path of tight knots, their volumes can only be calculated numerically. We can then calculate or estimate the volume and therefore the energy for a corresponding physical system. As with knots, the volumes of topologically non-trivial tight braids (those where the elements are woven together) can only be found approximately. While the simple helically twisted braid has a volume that depends on the pitch angle which can potentially be adjusted by experimental conditions, tight knots and links have no such adjustable parameter.

Let us begin with a discussion of tight links of flux in electromagnetic plasma. Movement of fluids can exhibit topological properties. Interrelation between hydro- and magnetic dynamics may cause magnetic fields, to exhibit topological properties as well. For example, for a perfectly conducting fluid, the (abelian) magnetic helicity $\mathcal{H}=\int d^{3} x \epsilon^{i j k} A_{i} \partial_{j} A_{k}$ is an invariant of the motion [10], and this quantity can be interpreted in terms of knottedness of magnetic flux lines [11]. (The helicity for two linked flux tubes with fluxes $\Phi_{1}$ and $\Phi_{2}$ is $\mathcal{H}=2 n \Phi_{1} \Phi_{2}$, where $n$ is the Gauss linking number of the two tubes. It is straightforward to generalize this to the case of linked and/or self-linked thick flux tubes.) A perfectly conducting, non-viscous, incompressible fluid relaxes to a state of magnetic equilibrium without a change in topology [1] . 
The system approaches a state of magnetic equilibrium by decreasing its magnetic energy by contraction of the magnetic field lines. In the case of trivial topology, closed curves contract to a point without crossing each other. The relaxation eventually leads to a state with zero fields (vacuum). If, however, the topology of the initial magnetic fields is non-trivial, the relaxation stops when flux tubes are tightly knotted or linked. This happens because the "freeze-in" condition forces topological restrictions on possible changes in field configurations and so any initial knots and links of field lines remain topologically unchanged during relaxation. The energy of a final (equilibrium) state is determined by topology.

By analogy with the abelian case, for a conserved nonabelian helicity [12], we choose the corresponding expression for helicity with topological properties, $\mathcal{H}=$ $\int_{V}\left(A d A+\frac{2}{3} A^{3}\right)$. In a perfectly conductive relativistic non-abelian plasma, the electric field vanishes in the local frame moving with the plasma. Details are analogous to the abelian case. With these facts in mind, we are now in position to ask if one could hope to find knotted/linked flux tubes in a physical system.

For several reasons we believe the ideal physical system in which to discover and study tight knots and links is Quantum Chromodynamics (QCD). These include: (1) QCD is a solidly based part of the standard model of particle physics, and much about color confinement and the quark model is already well understood in this context, making much previous work transferable to the problem of tightly knotted flux tubes in this theory. (2) Unlike plasmas, fluids or condensed matter systems where flux tubes are excitations of some media with many parameters that could hide universal behavior, flux knots in QCD can exist in the vacuum. Thus continuum states are absent and there are no media parameters to vary and obscure the universality. Hence, the results in QCD can be far less ambiguous. (3) The hadronic energy spectrum has been measured over a large range of energies (140 $\mathrm{MeV}$ to $10 \mathrm{GeV}$ ) and already many hundreds of states are known. We expect that among these, a few dozen can be classified as tightly knotted/linked flux tubes states. These states must have no valance quarks (i.e., no flavor quantum numbers) in order to be classified as glueballs. (4) Knotted solitons in QFT are already known to exist. (5) One can efficiently search for new glueball states at accelerators. (Also, data from older experiments still exist and can be reanalyzed to check the predictions of new states described below.)

Consider a hadronic collision that produces some number of baryons and mesons plus a gluonic state in the form of a closed QCD flux tube (or a set of tubes). From an initial state, the fields in the flux tubes quickly relax to an equilibrium configuration, which is topologically equivalent to the initial state. (We assume topological quantum numbers are conserved during this rapid process.) The relaxation proceeds through minimization of the field energy. Flux conservation and energy minimization force the fields to be homogeneous across the tube cross sections. This process occurs via shrinking the tube length, and halts to form a "tight" knot or link. The radial scale will be set by $\Lambda_{\mathrm{QCD}}^{-1}$. The energy of the final state depends only on the topology of the initial state and can be estimated as follows. An arbitrarily knotted tube of radius $a$ and length $l$ has the volume $\pi a^{2} l$. Using conservation of flux $\Phi_{E}$, the energy becomes $\propto l\left(\operatorname{tr} \Phi_{E}^{2}\right) /\left(\pi a^{2}\right)$. Fixing the radius of the tube (to be proportional to $\Lambda_{\mathrm{QCD}}^{-1}$ ), we find that the energy is proportional to the length $l$. The dimensionless ratio $\varepsilon(K)=l /(2 a)$ is a topological invariant and the simplest definition of the "knot energy" [13], and can be used to fit the correspondence between knot/link energies and glueball masses.

In our model, the chromoelectric fields $F_{0 i}$ are confined to knotted/linked tubes. After an initial time evolution, the system reaches a static equilibrium state which is described by the energy density $\mathcal{E}_{E}=\frac{1}{2} \operatorname{tr} F_{0 i} F^{0 i}-V$. Similar to the bag model, we have included a constant potential energy $V$ needed to keep the tubes at a fixed cross-section. The chromoelectric flux $\Phi_{E}$ is conserved and we assume flux tubes carry one flux quantum. To account for conservation of the flux, we add the term $\operatorname{tr} \lambda\left\{\Phi_{E} /\left(\pi a^{2}\right)-n^{i} F_{0 i}\right\}$ to the energy density, where $n^{i}$ is the normal vector to a section of the tube and $\lambda$ is a Lagrange multiplier. The energy density should be constant under variations of the degrees of freedom, the gauge potentials $A_{\mu}$. This leads to a constant field solution, $F_{0 i}=\left(\Phi_{E} / \pi a^{2}\right) n_{i}$. With this solution, the energy is positive and proportional to $l$ and thus the minimum of the energy is achieved by shortening $l$, i.e., tightening the knot.

Lattice calculations, QCD sum rules, electric flux tube models, and constituent glue models agree that the lightest non $-q \bar{q}$ states are glueballs with quantum numbers $J^{++}=0^{++}$and $2^{++}$[7]. We will model all $J^{++}$states (i.e., all $f_{J}$ and $f_{J}^{\prime}$ states listed by the PDG [7]), some of which will be identified with rotational excitations, as knotted/linked chromoelectric QCD flux tubes. We proceed to identify knotted and linked QCD flux tubes with glueballs, where we include all $f_{J}$ and $f_{J}^{\prime}$ states. The lightest candidate is the $f_{0}(600)$, which we identify with the shortest knot/link, i.e., the $2_{1}^{2}$ link; the $f_{0}(980)$ is identified with the next shortest knot, the $3_{1}$ trefoil knot, and so forth. All knot and link energies have been calculated for states with energies less then $1680 \mathrm{MeV}$. Above $1680 \mathrm{MeV}$ the number of knots and links grows rapidly, and few of their energies have been calculated (see alternatively, Ref. [4]). However, we do find knot energies corresponding to all known $f_{J}$ and $f_{J}^{\prime}$ states, and so can make preliminary identifications in this region. Our detailed results are collected in Table [1 where we list $f_{J}$ and $f_{J}^{\prime}$ masses, our identifications of these states with knots and the corresponding knot energies. In Fig. 1 we compare the mass spectrum of $f_{J}$ states with the identified knot and link energies. Since errors for the knot energies in Ref. 2] were not reported, we conservatively assumed the error to be $1 \%$. A least 
TABLE I: Comparison between the glueball mass spectrum and knot energies.

\begin{tabular}{|c|c|c|c|c|}
\hline State & Mass & $K^{a}$ & $\varepsilon(K)^{b}$ & $E(G)^{c}$ \\
\hline$f_{0}(600)$ & $400-1200$ & $2_{1}^{2}$ & $12.6[4 \pi]$ & $768[766]$ \\
\hline$f_{0}(980)$ & $980 \pm 10$ & $3_{1}$ & 16.4 & 993 \\
\hline$f_{2}(1270)$ & $1275.4 \pm 1.2$ & $2_{1}^{2} * 0_{1}$ & {$[6 \pi+2]$} & {$[1256]$} \\
\hline \multirow[t]{2}{*}{$f_{1}(1285)$} & $1281.9 \pm 0.6$ & $4_{1}$ & 21.2 & 1277 \\
\hline & & $4_{1}^{2}$ & $(21.4)$ & (1289) \\
\hline$f_{1}(1420)$ & $1426.3 \pm 1.1$ & $5_{1}$ & 24.2 & 1454 \\
\hline$\left\{f_{2}(1430)\right.$ & $\approx 1430\}^{d}$ & $5_{1}$ & 24.2 & $1454+\delta^{\prime}$ \\
\hline$f_{0}(1370)$ & $1200-1500$ & $3_{1} * 0_{1}$ & $(24.7)$ & (1484) \\
\hline$f_{0}(1500)$ & $1507 \pm 5$ & $5_{2}$ & 24.9 & 1496 \\
\hline$\left\{f_{1}(1510)\right.$ & $1518 \pm 5\}$ & $5_{2}$ & 24.9 & $1496+\delta$ \\
\hline$f_{2}^{\prime}(1525)$ & $1525 \pm 5$ & $5_{2}$ & 24.9 & $1496+3 \delta$ \\
\hline$\left\{f_{2}(1565)\right.$ & $1546 \pm 12\}$ & $5_{1}^{2}$ & $(25.9)$ & $(1555)$ \\
\hline \multirow[t]{2}{*}{$f_{2}(1640)$} & $1638 \pm 6\}$ & $6_{3}^{3}$ & $((27.3))$ & $((1638))$ \\
\hline & & $\left(2_{1}^{2} * 0_{1}\right) * 0_{1}^{e}$ & {$[8 \pi+3]$} & {$[1686]^{f}$} \\
\hline \multirow[t]{10}{*}{$f_{0}(1710)$} & $1713 \pm 6$ & $6_{2}^{3}$ & $((28.6))$ & $((1714))$ \\
\hline & & $3_{1} \# 3_{1}^{*}$ & $28.9(30.5)$ & $1732(1827)$ \\
\hline & & $3_{1} \# 3_{1}$ & $29.1(30.5)$ & $1744(1827)$ \\
\hline & & $2_{1}^{2} * 2_{1}^{2}$ & {$[8 \pi+4]$} & {$[1745]$} \\
\hline & & $6_{2}$ & 29.2 & 1750 \\
\hline & & $6_{1}$ & 29.3 & 1756 \\
\hline & & 63 & 30.5 & 1827 \\
\hline & & $7_{1}$ & 30.9 & 1850 \\
\hline & & $8_{19}$ & 31.0 & 1856 \\
\hline & & $8_{20}$ & 32.7 & 1957 \\
\hline$f_{2}(2010)$ & $2011_{-80}^{+60}$ & $7_{2}$ & 33.2 & 1986 \\
\hline \multirow[t]{3}{*}{$f_{4}(2050)$} & $2025 \pm 8$ & $8_{21}$ & 33.9 & 2028 \\
\hline & & $8_{1}$ & 37.0 & 2211 \\
\hline & & $10_{161,162}$ & 37.6 & 2247 \\
\hline$f_{2}(2300)$ & $2297 \pm 28$ & $8_{18}, 9_{1}$ & 38.3 & 2288 \\
\hline \multirow[t]{3}{*}{$f_{2}(2340)$} & $2339 \pm 60$ & $9_{2}$ & 40.0 & 2389 \\
\hline & & $10_{1}$ & 44.8 & 2672 \\
\hline & & $11_{1}$ & 47.0 & 2802 \\
\hline
\end{tabular}

${ }^{a}$ Notation $n_{k}^{l}$ means a link of $l$ components with $n$ crossings, and occurring in the standard table of links (see e.g. [15]) on the $k^{\text {th }}$ place. $K \# K^{\prime}$ stands for the knot product (connected sum) of knots $K$ and $K^{\prime}$ and $K * K^{\prime}$ is the link of the knots $K$ and $K^{\prime}$.

${ }^{b}$ Values are from [2] except for our exact calculations of $2_{1}^{2}, 2_{1}^{2} * 0_{1}$, and $\left(2_{1}^{2} * 0_{1}\right) * 0_{1}$ in square brackets, our analytic estimates given in parentheses, and our rough estimates given in double parentheses.

${ }^{c} E(G)$ is obtained from $\varepsilon(K)$ using the fit in Figure 1

${ }^{d}$ States in braces are not in the Particle Data Group (PDG) summary tables.

${ }^{e}$ This is the link product that is not $2_{1}^{2} * 2_{1}^{2}$.

${ }^{f}$ Resonances have been seen in this region, but are unconfirmed [7].

squares fit to the most reliable data (below $1680 \mathrm{MeV}$ ) gives $E(G)=(23.4 \pm 46.1)+(59.1 \pm 2.1) \varepsilon(K) \quad[\mathrm{MeV}]$, with $\chi^{2}=9.1$. The data used in this fit is the first seven $f_{J}$ states (filled circles in Fig. (1) in the PDG sum- mary tables. Inclusion of the remaining seven (nonexcitation) states (unfilled circles in Fig. (1) in Table — where either the glueball or knot energies are less reliable, does not significantly alter the fit and leads to 


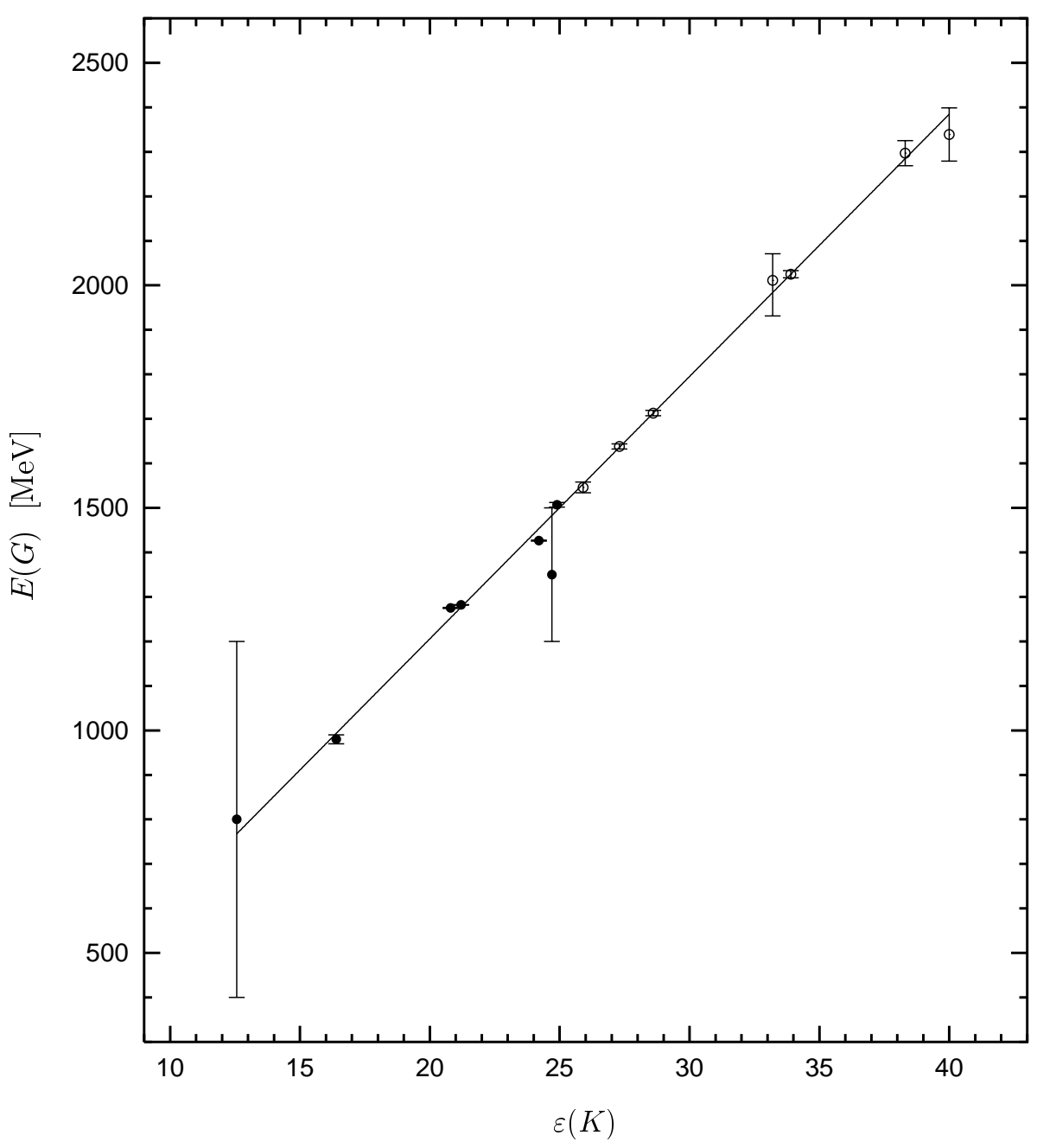

FIG. 1: Relationship between the glueball spectrum $E(G)$ and knot energies $\varepsilon(K)$. Each point in this figure represents a glueball identified with a knot or link. The straight line is our model and is drawn for the fit $E(G)=(23.4 \pm 46.1)+(59.1 \pm$ 2.1) $\varepsilon(K) \quad[\mathrm{MeV}]$

$E(G)=(26.9 \pm 24.9)+(58.9 \pm 1.0) \varepsilon(K) \quad[\mathrm{MeV}]$, with $\chi^{2}=10.1$. Both fits are in good agreement with our model, where $E(G)$ is proportional to $\varepsilon(K)$. Better HEP data and the calculation of more knot energies will provide further tests of the model and improve the high mass identification.

Knot complexity can be reduced (or increased) by unknotting (knotting) operations. In terms of flux tubes, these moves are equivalent to reconnection events. Hence, a metastable glueball may decay via reconnection. Once all topological charge is lost, metastability is lost, and the decay proceeds to completion. Two other glueball decay processes are: flux tube (string) breaking, which favors large decay widths for configurations with long flux tube components; and quantum fluctuations that unlink flux tubes, which tends to broaden states with short flux tube components. As yet we are not able to go beyond providing a phenomenological fit to these qualitative observations 5], but hope to be able to do so in the future.
In conclusion, let us return to continuum physics and consider a slab of material that can support flux tubes. We have in mind a super-fluid or superconductor, but are not limited to these possibilities. Assume further that the flux tubes carry one and only one unit of flux. Next consider manipulating these flux tubes. For instance, consider a hypothetical superconductor where the flux tubes are pinned at the bottom of the slab, say by being attracted to the poles of some magnetic material, and at the top of the slab they are each associated with the pole of a movable permanent magnet, perhaps a magnetic whisker, or fine solenoid. Assuming the tubes have time to relax to tight configurations, the energy released should correspond to the universal energy spectrum described above. Another collection of physical systems of potential interest are the atomic Bose-Einstein condensates. For example, laser stirring of dilute ${ }^{87} \mathrm{Rb}$ atoms at $80 \mathrm{nK}$ has produced vortices [14], which could lead to knots and links.

This work is supported in part by U.S. DoE grant 
\# DE-FG05-85ER40226.

[1] H. K. Moffatt, J. Fluid Mech. 159, 359 (1985).

[2] V. Katritch, et al., Nature 384, 142 (1996); V. Katritch, et al., Nature 388, 148 (1997).

[3] K. Millett and E. Rawdon, J. of Comp. Phys. 186, 426 (2003).

[4] R. V. Buniy, T. W. Kephart, M. Piatek and E. Rawdon, in preparation.

[5] R. V. Buniy and T. W. Kephart, Phys. Lett. B 576, 127 (2003).

[6] R. V. Buniy and T. W. Kephart, in combined Proceedings of "Knot Theory and its Applications," AMS Western Section Meeting (San Francisco, CA, May 2003), and "Knots, Random Walks and Biomolecules," (Les Diablerets, Switzerland, July 2003), to appear in the "Series on Knots and Everything," eds. J. Calvo, K. Millett, E. Rawdon, and A. Stasiak, World Scientific.

[7] K. Hagiwara et al., Phys. Rev. D 66, 010001 (2002).
[8] H. Weyl, Am. J. Math. 61, 461 (1939).

[9] A. Gray, Tubes, Addison-Wesley, 1990.

[10] L. Woltier, Proc. Nat. Acad. Sci. 44, 489 (1958).

[11] H. K. Moffatt, J. Fluid Mech. 35, 117 (1969).

[12] R. Jackiw, V. P. Nair, and So-Young Pi, Phys. Rev. D 62, 085018 (2000).

[13] H. K. Moffatt, Nature 347, 367 (1990); S. G. Whittington, D. W. Sumners, and T. Lodge, editors, Topology and Geometry in Polymer Science, Springer, 1998; R. A. Litherland, J. Simon, O. Durumeric, and E. Rawdon, Topology Appl. 91, 233 (1999); G. Buck and J. Simon, Topology Appl. 91, 245 (1999).

[14] M. Matthews at al., Phys. Rev. Lett. 83, 2498 (1999); K. Madison at al., Phys. Rev. Lett. 84, 806 (2000).

[15] D. Rolfsen, Knots and Links, Publish or Perish, 1990. 